\title{
INFLUENCE OF MATERIAL DAMPING ON THE RESPONSE OF UNDERGROUND STRUCTURES SUBJECTED TO EARTHQUAKE
}

\author{
Veronika Pavelcová, Tereza Poklopová, Michal Šejnoha*, \\ TOMÁŠ JANDA
}

\author{
Czech Technical University in Prague, Faculty of Civil Engineering, Department of Mechanics, Thákurova 7 , \\ 16629 Prague 6, Czech Republic \\ * corresponding author: sejnom@fsv.cvut.cz
}

\begin{abstract}
The paper describes a finite element simulation of the response of a real underground structure subjected to earthquake using GEO5 FEM program. It concentrates on the influence of material damping with respect to a specific type of boundary condition prescribed at the bottom of the analyzed domain. It is seen that considering material damping is inevitable particularly in case of so called fixed boundary conditions to arrive at meaningful results. This is demonstrated on an artificial earthquake generated according to a design spectrum defined in Eurocode 8. A viscous damping ratio combined with the results of eigenvalue analysis is used to derive parameters of Rayleigh damping for three specific scenarios promoting the approach based on the lowest natural frequency as sufficiently accurate for the present task.
\end{abstract}

KEYwORDs: Earthquake, tunnel, material damping, accelerogram, finite element method, absorbing boundary, fixed boundary.

\section{INTRODUCTION}

Numerical modeling of geotechnical structures subjected to earthquake typically distinguishes between damping associated with the application of radiation boundary condition [1] and intrinsic material damping associated with the energy dissipation within the material caused by various mechanisms including interparticle sliding and friction and pore fluid viscosity [2]. The former type of damping has been addressed in detailed in [3, 4]. The present contribution is therefore an extension of our previous work with emphasis on the effect of material damping.

Although valid for a single degree of freedom the complex structural analysis usually grounds on the knowledge of a single value damping ratio. Such a parameter is typically estimated experimentally from a hysteric stress-strain loop as the energy loss in a cycle [5]. While some new measurement technique arise [6] to eliminate uncertainty in the determination of geometric damping thus increasing reliability in the damping ratio measurement, the determination of damping ratio for all mode shapes is still far from trivial. This is why most practical applications rely on a single value damping ratio even in case of multi degree of freedom tasks combined with the concept of Rayleigh proportional damping [7]. This computational strategy is adopted also in the present study focused on the effect of material damping in combination with various types of boundary conditions introduced at the bottom of the computational model.

The remainder of the paper is organized as follows. Starting with a theoretical background in Section 2 we continue in Section 3 with the definition of material damping. The theoretical part of the paper is finalized in Section 4 addressing the construction of artificial accelerograms. Details of the computational models including the finite element mesh refinement are provided in Section 5. The overview of computational results starts in Section 6 with modal analysis to provide data for the derivation of parameters of Rayleigh damping. The results of free field (FF) analysis are presented in Section 7 followed by the summary of the essential outcomes delivered by two-dimensional (2D) analyses reflecting the presence of a tunnel.

\section{Governing EQUATIONS}

A detailed derivation of the set of governing equations driving the earthquake analysis in geotechnical engineering can be found, e.g., in [1, 3, 8]. Therein, the formulation was limited to damping associated with absorbing boundary conditions.

When taking into account the material damping the discretized form of the equation motion with quiet boundary assumed at the bottom of the model becomes, see also Fig. 1

$$
\begin{array}{r}
\mathbf{M} \ddot{\boldsymbol{u}}_{R}+\mathbf{C} \dot{\boldsymbol{u}}_{R}+\boldsymbol{K} \boldsymbol{u}_{R}+\left.\mathbf{C}^{\mathrm{BB}} \dot{\mathbf{u}}_{R}\right|_{y=0}+\left.\mathbf{C}^{\mathrm{LB}} \dot{\mathbf{u}}_{R}\right|_{x=0, L} \\
=-\mathbf{M} \ddot{\boldsymbol{u}}_{I 0}-\mathbf{C} \dot{\boldsymbol{u}}_{I 0}+\left.\mathbf{C}^{\mathrm{BB}} \dot{\mathbf{u}}_{I 0}\right|_{y=0}+\left.\mathbf{C}^{\mathrm{LB}} \dot{\mathbf{u}}_{R}^{F F}\right|_{x=0, L} \\
-\left.\boldsymbol{R}_{\tau}\right|_{x=0}+\left.\boldsymbol{R}_{\tau}\right|_{x=L},
\end{array}
$$

assuming the total displacement $\boldsymbol{u}(\boldsymbol{x}, t)$ be split into a relative displacement $\boldsymbol{u}_{R}(\boldsymbol{x}, t)$ and the displacement $\boldsymbol{u}_{I 0}(t)$ applied to the whole domain such that

$$
\boldsymbol{u}(\boldsymbol{x}, t)=\boldsymbol{u}_{R}(\boldsymbol{x}, t)+\boldsymbol{u}_{I 0}(t) .
$$

In Eq. (1), $\mathbf{M}, \mathbf{K}$ are the mass and stiffness matrices of the domain, respectively. The damping matrix $\mathbf{C}$ 


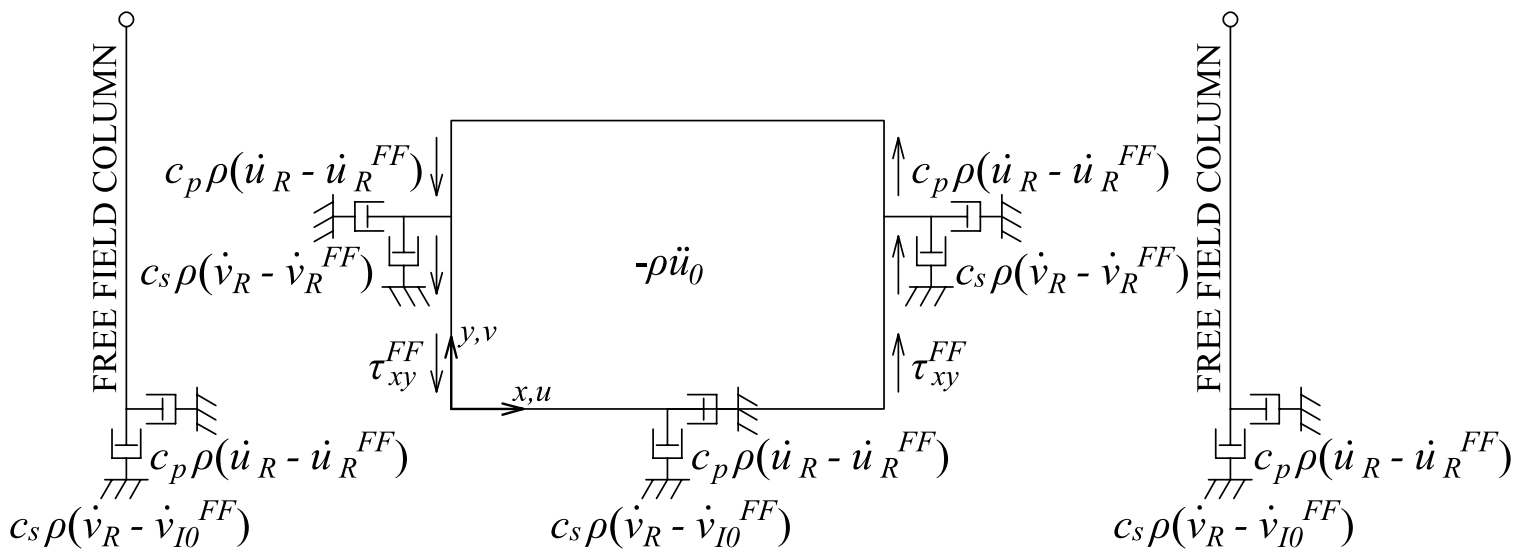

\section{ABSORBING BOUNDARY CONDITION $u(y, t)=u_{I 0}(t)+u_{R}(y, t)$}

Figure 1. Boundary and loading conditions for both FF and 2D analysis.

corresponds to material damping only, while matrices $\mathbf{C}^{\mathrm{BB}}$ and $\mathbf{C}^{\mathrm{LB}}$ reflect the effect of artificial viscous dampers applied along the absorbing boundaries (the superscripts BB and LB identify the bottom and lateral boundaries; henceforth the absorbing BB boundary will be called the quiet boundary and denoted as QBB whereas the notation FBB will be adopted to identify the so called fixed boundary [3, 8]). Finally, the vector $\boldsymbol{R}_{\tau}$ stores the nodal forces associated with the prescribed boundary shear stress.

Recall that in case of QBB the vector $\boldsymbol{u}_{I 0}(t)$ stands for the incoming wave only. Should the top soil be supported by an infinitely stiff bedrock we may introduce at the bottom boundary the FBB conditions suggesting a total reflection of the incoming wave back into the domain. The applied load than corresponds to the total motion $\boldsymbol{u}_{0}(t)$ so that

$$
\boldsymbol{u}(\boldsymbol{x}, t)=\boldsymbol{u}_{R}(\boldsymbol{x}, t)+\boldsymbol{u}_{0}(t),
$$

and Eq. (1) simplifies as

$$
\begin{array}{r}
\mathbf{M} \ddot{\boldsymbol{u}}_{R}+\mathbf{C} \dot{\boldsymbol{u}}_{R}+\boldsymbol{K} \boldsymbol{u}_{R}+\left.\mathbf{C}^{\mathrm{LB}} \dot{\mathbf{u}}_{R}\right|_{x=0, L} \\
=-\mathbf{M} \ddot{\boldsymbol{u}}_{0}-\mathbf{C} \dot{\boldsymbol{u}}_{0}+\left.\mathbf{C}^{\mathrm{LB}} \dot{\mathbf{u}}_{R}^{F F}\right|_{x=0, L} \\
-\left.\boldsymbol{R}_{\tau}\right|_{x=0}+\left.\boldsymbol{R}_{\tau}\right|_{x=L},
\end{array}
$$

For simplicity, the present study assumes the incoming wave be equal to one half of the total motion.

\section{RAYLEIGH DAMPING}

Material damping may have a strong impact on the response of an underground structure when subjected to earthquake. However, a physically supported formulation of damping matrix $\mathbf{C}$ is not an easy task mainly because of the lack of experimental evidence. The proportional Rayleigh damping is then a typical choice in most practical applications. In such a case, the damping matrix is expressed as a linear combination of the mass and stiffness matrices in the form, see e.g. [9, 10,

$$
\mathbf{C}=\alpha \mathbf{M}+\beta \mathbf{K} .
$$

Accepting Eq. (5) allows us to write the damped natural frequency $\omega_{i}^{d}$ associated with the $i$-th orthogonal mode shape $\phi_{i}$ of undamped system in terms of the viscous damping ratio $\xi_{i}$ and the $i$-th natural frequency of undamped system $\omega_{i}$

$$
2 \omega_{i}^{d}=2 \xi_{i} \omega_{i}=\phi_{i}^{\top} \mathbf{C} \phi_{i}=\alpha+\beta \omega_{i}^{2},
$$

because

$$
\phi_{i}^{\top} \mathbf{M} \phi_{i}=1, \phi_{i}^{\top} \mathbf{K} \phi_{i}=\omega_{i}^{2} .
$$

Thus if two viscous damping ratios $\xi_{i}$ are known for the pair of natural frequencies $\omega_{i}$, the values of parameters $\alpha, \beta$ readily follow from Eq. (6). However, most often only a single value of parameter $\xi$ is provided. Then, the hypothetical assumption of the first natural frequency being least damped is usually accepted. Therefore,

$$
\frac{d \xi}{d \omega}=\frac{1}{2}\left(-\frac{\alpha}{\omega^{2}}+\beta\right)=0 .
$$

Substituting $\omega=\omega_{1}$ into Eqs. (6) and (8) then gives

$$
\alpha=\xi_{1} \omega_{1}, \quad \beta=\frac{\xi_{1}}{\omega_{1}} .
$$

\section{Synthetic aCCElerograms}

A finite element analysis of geotechnical structures subjected to earthquake-induced loading requires specifying the time history of free field accelerations. Eurocode 8 [11] allows for using either recorded or artificial accelerograms. The artificial accelerograms have to be generated to match the elastic response spectrum defined within Eurocode 8. An example of such a spectrum used in the present study is plotted in Fig. 2

The algorithm for generating artificial accelerograms utilized in this paper is mostly inspired by the algorithm proposed in [12 and consists of the following steps: 


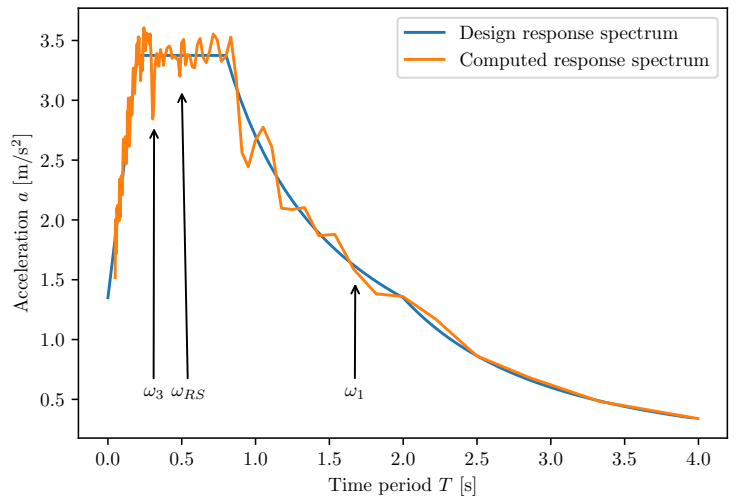

FiguRE 2. Design response spectrum, computed response spectrum, location of frequencies to calculate parameters of Rayleigh damping.

(1.) A Fourier spectrum with constant spectral amplitudes and random phases is generated.

(2.) Time history of acceleration is obtained from the spectrum by means of Fourier transform.

(3.) Elastic responses of a single degree-of-freedom systems with natural periods corresponding to frequencies used in the Fourier spectrum are computed for the time history.

(4.) Factors of the computed elastic response acceleration and the design response acceleration are evaluated.

(5.) The spectral amplitudes of the original Fourier spectrum are divided by the factors while the phases are kept.

(6.) Steps 2-5 are repeated for the updated Fourier spectrum until the computed elastic response spectrum matches the design elastic response spectrum within $10 \%$ relative error, see Fig. 2

Although the generated signal matches the target elastic response spectrum, it is stationary and lacks the characteristic stages of a typical earthquake signal. To take the rise, the strong motion and the decay periods into account, the amplitude of the stationary signal is modulated by an envelope function [13]

$$
E(t)=a t^{b} \exp (-c t)
$$

with parameters

$$
\begin{aligned}
b & =\frac{-\varepsilon \ln \eta}{1+\varepsilon(\ln \varepsilon-1)}, \\
c & =\frac{b}{\varepsilon T_{w}} \\
a & =\left(\frac{\exp (1)}{\varepsilon T_{w}}\right)^{b},
\end{aligned}
$$

where $T_{w}$ denotes the duration of earthquake. Peak of the envelope function occurs at fraction $\varepsilon$ of the earthquake duration and the original amplitude is reduced to $\eta$ fraction at time $T_{W}$. An example of

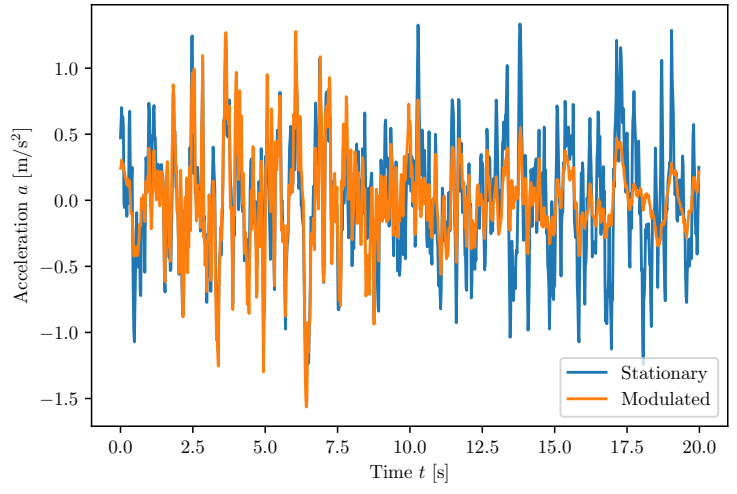

FiguRE 3. An example of stationary (original) and modulated (with rise, strong motion and decline stages) accelerograms.

stationary and modulated synthetic accelerogram is shown in Figure 3

\section{MODEL DESCRIPTION}

Two computational models were created to demonstrate the effect of material damping in relation to the selected boundary conditions applied at the bottom of the domain. The first model in Fig. 4(a) consists of a soft layer supported by an infinite layer of a relatively stiff bedrock. Truncating the model within the rock layer thus requires application of absorbing boundary conditions ensuring that the incoming wave is fully damped and not reflected back into the domain. The second model in Fig. 4(b) assumes in turn the bedrock be infinitely stiff thus replacing this layer with the fixed boundary. Both layers are assumed linearly elastic with material properties listed in Table 1 .

\begin{tabular}{lccc}
\hline Soil & & & \\
\hline Density & $\rho$ & 1960 & {$\left[\mathrm{~kg} / \mathrm{m}^{3}\right]$} \\
Poisson ratio & $\nu$ & 0.40 & {$[-]$} \\
Elastic modulus & $E$ & 80.00 & {$[\mathrm{MPa}]$} \\
\hline Rock & & & \\
\hline Density & $\rho$ & 2170 & {$\left[\mathrm{~kg} / \mathrm{m}^{3}\right]$} \\
Poisson ratio & $\nu$ & 0.30 & {$[-]$} \\
Elastic modulus & $E$ & 630.00 & {$[\mathrm{MPa}]$} \\
\hline \multicolumn{4}{c}{ TABLE 1. Material parameters. }
\end{tabular}

As in [3] the tunnel being $6 \mathrm{~m}$ in diameter is located in the soil layer at a depth of $37.35 \mathrm{~m}$. The tunnel lining is modeled by linearly elastic beam elements.

\section{RAYLEIGH DAMPING PARAMETERS}

As demonstrated in Section 3 , defining parameters $\alpha, \beta$ in Eq. (5) requires the knowledge of a pair of natural frequencies, Eq. (6), or at least of the first natural frequency $\omega_{1}$ should Eq. $(9)$ be used. 


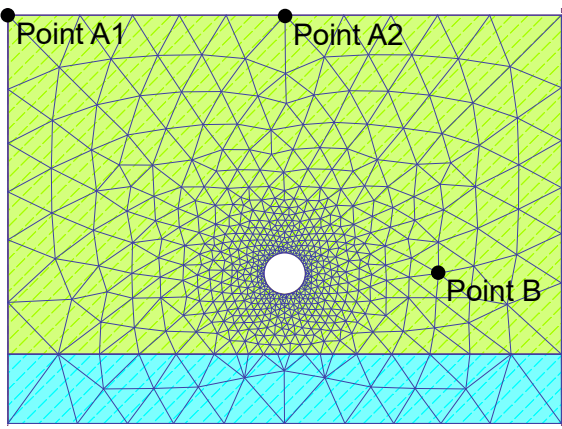

(a)
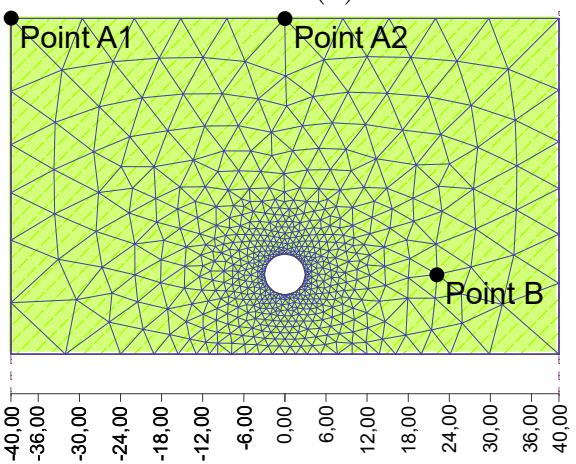

(b)

Figure 4. Computational model - a) quiet boundary conditions $(\mathrm{QBB})$, b) fixed boundary conditions (FBB).

Eurocode 8 suggests proportional damping with a single value of $\xi=0.05$ (proportional damping of $5 \%$ ). Thus the way of calculating parameters $\alpha, \beta$ determines, how much will individual natural frequencies be damped. The following three options are examined in this study

- Type 1: The first natural frequency is assumed to be the least damped frequency.

- Type 2: The least damped frequency domain is between the first and the third natural frequency. This option results from the recommendation provided in the PLAXIS user manual [14.

- Type 3: The least damped frequency domain is between the first natural frequency and the most dominant frequency $\omega_{R S}=12.5664 \mathrm{rad} / \mathrm{s}$ of the design response spectrum displayed in Fig. 2 .

The required natural frequencies were found from a standard eigenvalue analysis. The first three natural frequencies corresponding to the shear dominated mode shapes were extracted from the list of calculated frequencies based on the value of a significant modal participation factor [15] for the $x$ direction, recall the coordinate system displayed in Fig. 1. Three analyses in particular were carried out. The first one assumed the free field column (1DFF), the second one adopted the 2D model in Fig. 4(a) (2DT) and the third one considered the same model but for the state prior to excavation (2DI). In all calculations, the bottom boundary was fixed. In 2D models the lateral boundaries were additionally constrained in the $y$-direction to recover the mode shapes compatible with the free field column analysis. The results are stored in Table 2 The values in parentheses represent the actual order when considering all modes.

\begin{tabular}{lccc}
\hline & $\omega_{1}$ & $\omega_{2}$ & $\omega_{3}$ \\
\hline 1DFF & $3.7547(1)$ & $11.5307(3)$ & $20.1191(4)$ \\
2DT & $3.7481(1)$ & $11.4440(5)$ & $19.5180(10)$ \\
2DI & $3.7505(1)$ & $11.3844(5)$ & $19.4204(10)$ \\
\hline \multicolumn{5}{c}{ TABLE 2. Calculated natural frequencies. }
\end{tabular}

As can be seen, all analyses provided almost identical results. Thus to determine parameters $\alpha, \beta$ in Table 3 the results from 2DI analysis were employed.

\begin{tabular}{lcccc}
\hline Damping & $\omega$ & $\xi$ & $\alpha$ & $\beta$ \\
\hline Type 1 & $\omega_{1}$ & 0.05 & 0.1875 & 0.0133 \\
Type 1b & $\omega_{1}$ & 0.10 & 0.3750 & 0.0266 \\
Type 2 & $\omega_{1}+\omega_{3}$ & 0.05 & 0.3143 & 0.0043 \\
Type 3 & $\omega_{1}+\omega_{R S}$ & 0.05 & 0.2888 & 0.0061 \\
\hline
\end{tabular}

TABLE 3. Rayleigh damping parameters.

Figure 5 shows how the natural frequencies are damped for the three types of damping with $\xi=0.05$.

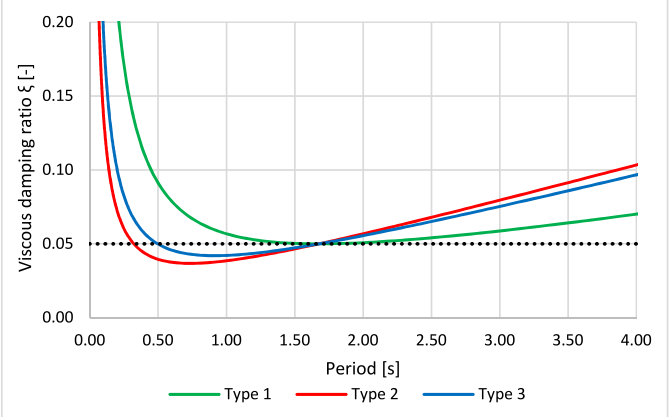

Figure 5. Viscous damping ratio as a function of the natural period (frequency).

\section{1D FREE FIELD ANALYSIS}

The influence of material damping was first addressed in the light of one-dimensional free-field column analysis. This is in fact also the first step in a twodimensional analysis needed in generating the time variation of free-field velocities and boundary shear stresses. It also allows us to test the influence of finite element mesh, which is in this case quite non-uniform as seen in Fig. 4. The 1DFF mesh corresponds to the refinement along the edges of the $2 \mathrm{D}$ domains and certainly is not optimal neither from the material, nor from the integration time step point view, which was 
set to $0.01 \mathrm{~s}$ in accordance with the adopted acceleration record. Only the soil layer was assumed to be damped. All the results presented in Figs. 6- 9 show variation of a relative displacement along the $x$-direction $u_{R x}$ observed on the terrain (Point A1).

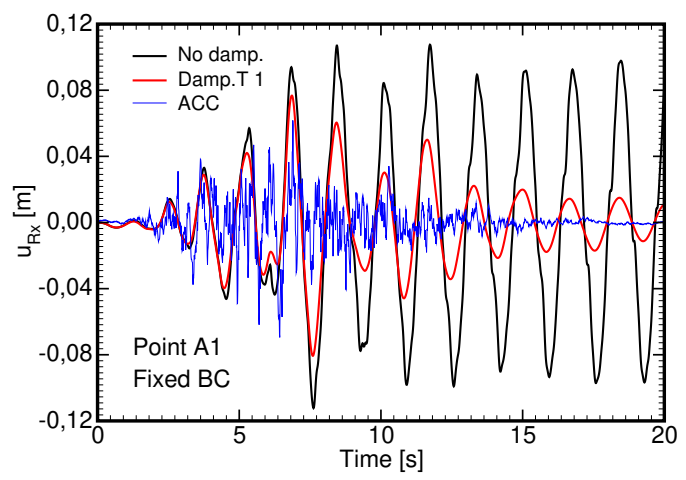

Figure 6. Time variation of horizontal displacement $u_{R x}$ monitored at Point A1. Blue line is the scaled variation of the prescribed acceleration.

Figure 6 compares variation of damped and undamped displacement $u_{R x}$ considering the fixed boundary conditions, Fig. 4(b). The scaled acceleration is added just to illustrate the time interval of strong motion. The results suggest that appropriate damping is absolutely essential in case of fixed boundary, because in undamped model the vertically propagating shear waves reflect not only from the terrain free boundary but also from the fixed bottom boundary. Therefore, in the undamped model the motion does not cease even after the input signal diminishes.

The influence of the selected type of damping, recall Section 6 and Table 3 is examined in Figs. 7 and 8 While some differences in the model response can be observed in Fig. 8 in the case of QBB, the results plotted in Fig. 7 considering FBB suggest almost negligible effect of the type of damping, at least in the present example. It is also seen in Fig. 8 that unlike the fixed boundary, the influence of damping in the case of QBB is far less pronounced. This is attributed to the fact that the downward traveling wave is intentionally damped at the bottom boundary having the same effect as allowing the downward traveling waves to leave the examined model at the truncated boundary, so the motion eventually stops even without material damping.

To directly compare the results derived for FBB and QBB we first plot in Fig. 9 the time variation of the horizontal displacement $u_{R x}$ monitored at various points to see that the displacement at the interface of the two materials practically coincides with the motion monitored at the bottom boundary. This indicates that the vertically propagating wave travels very fast through the rock layer so the difference between the two models dimensions in Fig. 4 can safely be neglected. This comparison of the response of damped model with FBB and undamped model with QBB is plotted in Fig. 10.

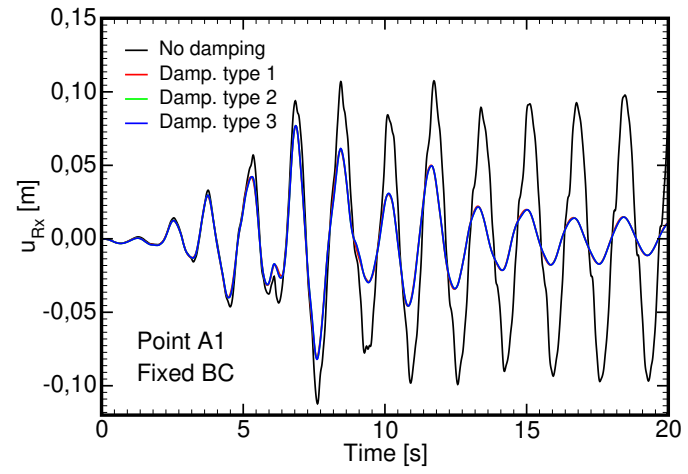

Figure 7. Influence of the type damping - FBB.

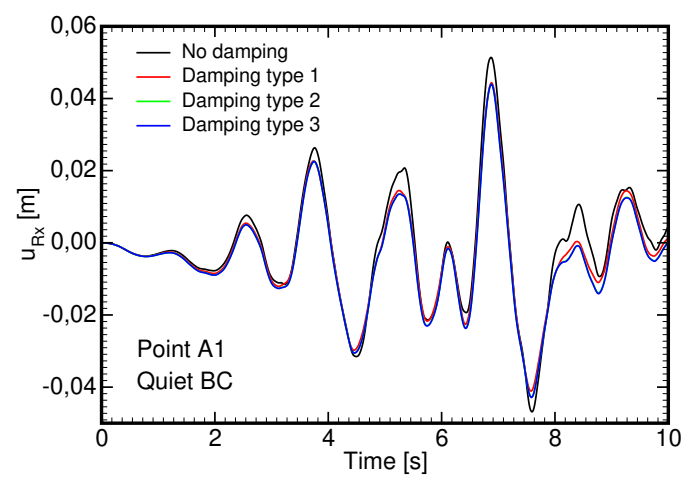

Figure 8. Influence of the type damping - QBB.

It can be observed that even with $5 \%$ damping, the model with fixed bottom boundary yields nonnegligible displacements even beyond $20 \mathrm{~s}$ of monitoring where the prescribed motion comes to zero. The figure also shows the importance of correctly prescribing only the incoming wave for the model with QBB. Adopting the total motion would yield the displacement at the terrain exceeding the motion found in the model with FBB, which is unrealistic.

\section{2D ANALYSIS OF TUNNEL}

Taking into account the results discussed in the previous section the $2 \mathrm{D}$ analyses were limited to the QBB model in Fig. 4(a) assuming no damping, while only the damping type 1 was considered when examining the FBB model in Fig. 4(b). For both models, the horizontal displacements plotted in Fig. 11 were monitored at points A2 and B.

Similar conclusions to what has been observed in Section 7 can be drawn here suggesting again the need for material damping with fixed boundary. Because choosing the particular value of damping ratio has mostly no rigorous support, we compared the results pertinent to $5 \%$ of damping to those derived for $10 \%$ of damping. Again the 5\% of damping still overestimates the displacements at the end of earthquake, while the results found for $10 \%$ of damping resemble the response of QBB model at least at the terrain boundary. In the vicinity of tunnel lining, the motion is, however, overdamped. 


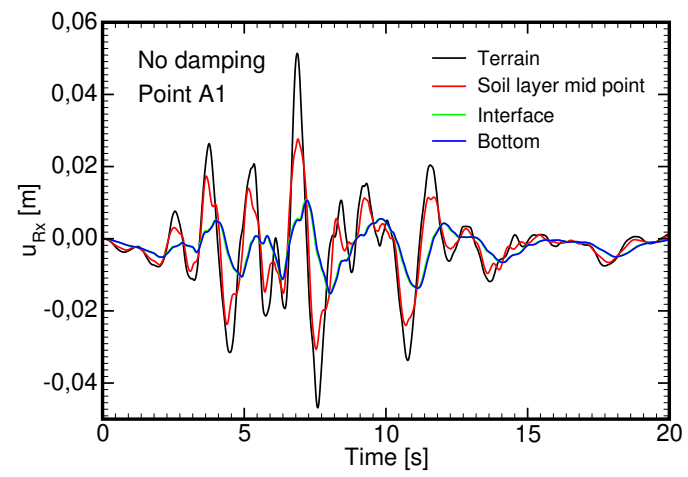

Figure 9. Time variation of horizontal displacement $u_{R x}$ monitored at various points - QBB.

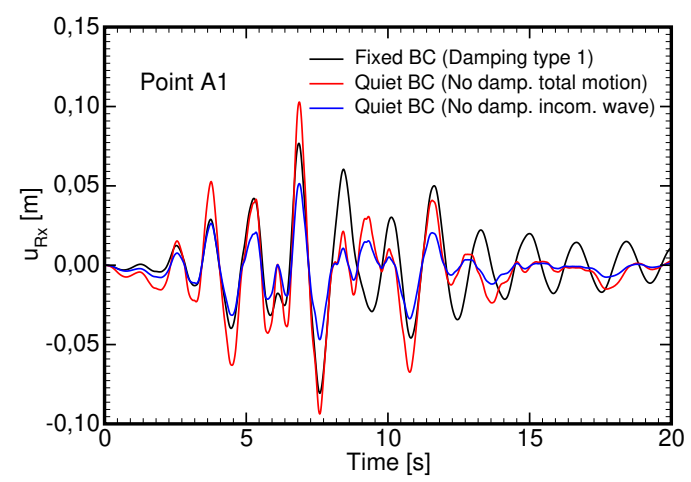

FIGURE 10. Comparison of damped model with FBB and undamped model with QBB.

A smooth variation of damped displacements also suggests that particularly high frequencies of the motion are damped, which agrees well with Fig. 5 . Another observation follows from a close inspection of Figs. 10 and 11(a) showing only a minor difference between the response provided by $1 \mathrm{DFF}$ and $2 \mathrm{DT}$ analyses, mainly because of a relatively stiff lining. This thus supports the results obtained already from modal analysis in Section 6

\section{Conclusions}

The present paper examined the influence of material damping in earthquake analysis particularly with reference to the boundary conditions prescribed at the bottom boundary of the model. The following conclusions can be proposed:

- Considering material damping in case of QBB has only a minor effect on the predicted response.

- On the other hand, the FBB model should not be considered without material damping as rather erroneous results can be obtained.

- The way of determining the Rayleigh damping parameters has, at least in the present study, a relatively minor effect.

\section{ACKNOWLEDGEMENTS}

The support provided by the SGS project No. SGS19/032/OHK1/1T/11 and the TAČR project No.

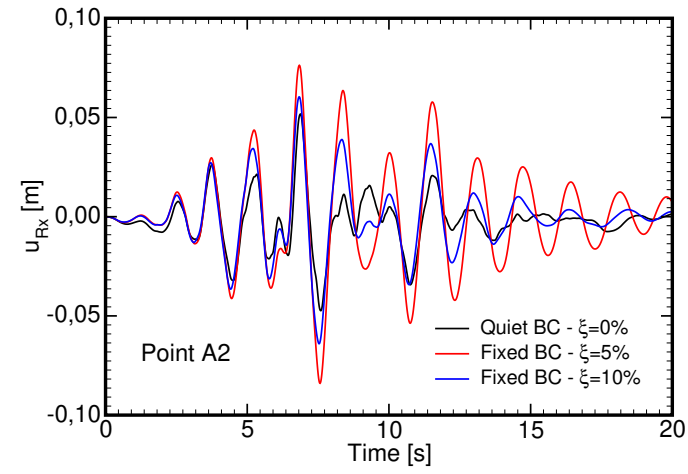

(a)

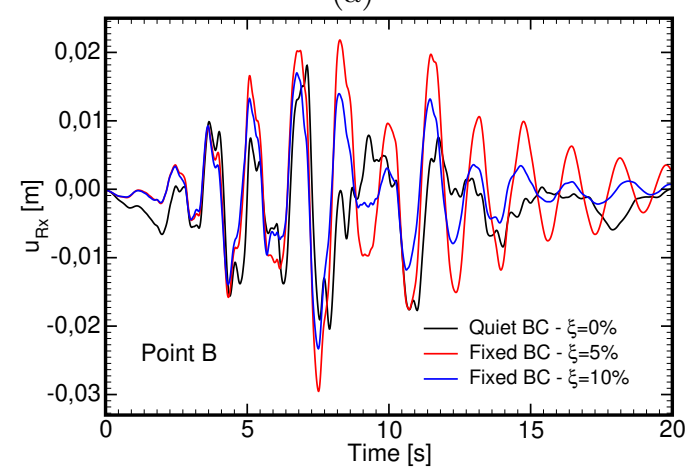

(b)

Figure 11. Time variation of relative displacement at selected points during the period of strong motion: a) point $\mathrm{A}, \mathrm{b}$ ) point $\mathrm{B}$.

TE01020168 is gratefully acknowledged.

\section{REFERENCES}

[1] O. Zinkiewicz, N. Bicanic, F. Shen. Generalized Smith boundary - a transmitting boundary for dynamic computations, vol. 207. INNME Swansea, 1986. DOI:10.1007/978-3-7091-2828-2_3.

[2] A. Ashmawy, R. Salgado, S. Guha, V. Drnevich. Soil damping and its use in dynamic analyses. In Third International Conferences on Recent Advances in Geotechnical Earthquake Engineering and Soil Dynamics. Missouri University of Science and Technology, 1995. Paper No. 1.13.

[3] V. Pavelcová, T. Poklopová, T. Janda, M. Šejnoha. The influence of boundary conditions on the response of underground structures subjected to earthquake. Acta Polytechnica, CTU Proceedings 15:74-80, 2018. DOI:10.14311/APP.2018.15.0074.

[4] J. Pruška, M. Šejnoha, T. Janda, et al. Analysis of underground structures subjected to earthquake using finite element method. In 14 th International Conference entitled "Underground Construction Prague 2019". ITA-AITES Czech Tunnelling Association, 2019. June 3-5, Prague.

[5] I. Towhata. Geotechnical Earthquake Engineering. Springer-Verlag Berlin Heidelberg, 2008.

[6] S. Badsar, M. Schevenels, W. Haegeman, et al. Determination of the material damping ratio in the soil from SASW tests using the half-power bandwidth method. Geophysical Journal International 182:14931508, 2010. DOI:10.1111/j.1365-246X.2010.04690.x 
[7] A. Zidan. Modeling of soil damping for seismic ground response by nonlinear finite element analysis. In Third International Conference on Advances in Civil and Structural Engineering - CSE. Institute of Research Engineers and Doctors, USA, 2015.

[8] V. Pavelcová. Evaluation of real underground structure subjected to earthquake - fully dynamic analysis.

Bachalor's thesis, Czech Technical University in Prague, Faculty of Civil Engineering, 2018. In Czech.

[9] O. Řřricha, Z. Bittnar. Metoda konečných prvkův dynamice konstrukcí. Nakladatelství technické literatury, 1981.

[10] Z. Bittnar, J. Šejnoha. Numerical methods in structural engineering. ASCE Press, 1996.

[11] Comité Européen de Normalisation. Eurocode 8: Design of structures for earthquake resistance, 2004.
[12] A. Kumar. Software for generation of spectrum compatible time history. In Proceedings of 13th World Conference on Earthquake Engineering. Canadian Association for Earthquake Engineering, 2004.

[13] D. Boore. Stochastic simulation of high frequency ground motion based on seismological models of radiated spectra. Bulletin of Seismological Society of America 73(6):1865-1894, 1983.

[14] PLAXIS manual, http://www.plaxis.nl/.

[15] T. Irvine. Effective modal mass and modal participation factors Revision H, 2013. 